\title{
Modeling of Dissolution of Weakly Soluble Salts in the Presence of Complexing Agents
}

\author{
Artem I. Korobeinikov* and Sergey N. Kalyakin \\ Institute of Chemistry and Chemical Technology SB RAS \\ FRC "Krasnoyarsk Science Center SB RAS" \\ 50/24 Akademgorodok, Krasnoyarsk, 660036, Russia
}

Received 26.09.2018, received in revised form 21.12.2018, accepted 20.01.2019

\begin{abstract}
A mathematical algorithm for ionic equilibria modeling of dissolving weakly soluble salts in the presence of complexing agents or in an overage of an anion that form weakly soluble compounds in water solutions was developed. For numerical methods for calculation of chemical equilibria that involve ionic compounds a set of simplifications are formulated. This simplifications allows to significantly reduce the required computational operations while make it possible to preserve the functionality of numerical methods for solving such ionic equilibria. To verify the algorithm some systems that was studied experimentally were modelled. The maximum divergence between calculated and experimental data was 38\%. Some general problems of switch-over from the experimental determination to the mathematical modeling of ionic equilibria and sedimentation in solution were emphasized.
\end{abstract}

Keywords: mathematical modelling, computer program, numerical methods, weakly soluble compound, precipitate, dissolution, complex compounds.

Citation: Korobeinikov A.I., Kalyakin S.N. Modeling of dissolution of weakly soluble salts in the presence of complexing agents, J. Sib. Fed. Univ. Chem., 2019, 12(1), 87-100. DOI: 10.17516/1998-2836-0079.

(C) Siberian Federal University. All rights reserved

* Corresponding author E-mail address: artkor7771988@gmail.com 


\title{
Моделирование растворения малорастворимых солей в присутствии комплексообразователей
}

\author{
А.И. Коробейников, С.Н. Калякин \\ Институт химии и химической технологии СО РАН \\ ФИЦ «Красноярский научный иентр СО РАН» \\ Россия, 660036, Красноярск, Академгородок, 50/24
}

Разработан математический алгоритм моделирования ионных равновесий в водных растворах при растворении малорастворимых солей в присутствии комплексообразователей или в избытке аниона, образующего малорастворимые соединения. Для численных методов расчета химических равновесий с участием ионных соединений сформулирован ряд упрощений, которые при значительном уменьшении требуемых вычислительных операций позволяют сохранить функииональность численных методов для решения указанных ионных равновесий. Для верификации алгоритма было проведено моделирование некоторых систем, по которым имеются достоверные экспериментальные данные. Максимальное отклонение между расчетными и экспериментальными данными составило $38 \%$. Были отмечены некоторые общие проблемь перехода от экспериментального определения к математическому моделированию ионных равновесий и осадкообразования в растворе.

Ключевые слова: математическое моделирование, компьютерная программа, численные методы, малорастворимое соединение, осадок, растворение, комплексные соединения.

\section{Введение}

Задача математического моделирования химических равновесий с участием ионов в растворе представляется крайне сложной ввиду того, что любой подобный расчет представляет собой решение системы разнотипных уравнений, а именно, материального баланса (линейных), ионных равновесий (линейных после логарифмирования) и коэффициентов активности ионов (сложная зависимость от ионной силы в растворе, заряда иона и активности воды). Подобная структура получаемой системы уравнений приводит к тому, что она нерешаема аналитическим способом.

В настоящее время разработано несколько итерационных методов, которые позволяют находить решение ионных равновесий в водных растворах. В частности, метод С.Р. Бринкли (метод итераций Ньютона, модифицированный под решение ионных равновесий) состоит в том, что в пространстве концентраций так называемых независимых частиц (количество независимых частиц обычно равно количеству уравнений материального баланса в моделируемой системе, независимые частицы выбираются произвольным образом или по какому-либо алгоритму из числа возможных в системе частиц) выбирается вектор, который на каждой итерации меняет координату и направление в зависимости от результата на предыдущих итерациях, постепенно приближаясь к значениям концентраций, которые соответствуют решению исходной системы уравнений [1]. 
Тем не менее данный метод, как и другие итерационные методы, имеет ряд недостатков. К данным недостаткам следует отнести:

1. При применении метода простой итерации и аналогичных методов - экспоненциальный рост количества необходимых вычислительных операций при увеличении числа независимых частиц [2].

2. Ввиду того что пространство концентраций не является выпуклым, а теоретические концентрации разных частиц в одной системе могут составлять от молей на л до $10^{-50}$ моль/л и даже меньше при применении методов, использующих матрицу Якоби (пример - метод Ньютона), возникают следующие трудности:

- необходимость определять начальное приближение, близкое к истинному результату, при этом универсального алгоритма для определения не существует [3];

- сбои при выполнении программного кода в случае появления отрицательных значений концентраций на какой-либо итерации [3];

- появление при решении матричных уравнений серьезной программной погрешности вычисления в случае одновременного присутствия в матрице величин, которые отличаются на много порядков.

3. В случае, если решаемая система уравнений имеет не единственный экстремум (в качестве функции обычно принимается разность истинного и полученного на данной итерации материального баланса, под экстремумом тут понимается только минимум указанной разницы), что вполне вероятно при расчетах ионных равновесий в растворах для многокомпонентных и/или гетерофазных систем, то итерации могут «застрять» в области, содержащей локальный экстремум, не являющийся решением системы.

4. Итерационные методы для расчета ионных равновесий в растворах не позволяют включать в исходную систему уравнений какие-либо другие уравнения, кроме уравнений ионных равновесий и уравнений материального баланса, ввиду того что это приведет к чрезмерному росту числа локальных экстремумов. Это означает, что другие параметры, например ионная сила $(I)$ и коэффициент активности воды $\left(a_{w}\right)$, должны быть заданы заранее, хотя данные параметры (особенно $I$ ), в свою очередь, могут в значительной степени зависеть от ионного состава раствора.

Вследствие вышеописанной проблемы, несмотря на высокую степень развития вычислительной техники и численных методов, для решения подобных задач до сих пор не разработан универсальный и полностью автоматизированный программный продукт. При этом существует практика разработки узкоспециализированных расчетных методов и программного обеспечения для определения состава равновесных систем при решении задач описания и оптимизации отдельных технологических процессов. Данные методы разрабатываются с некоторыми допущениями, значительно упрощающими расчеты, но справедливыми лишь для данных конкретных условий.

В настоящее время в открытом доступе отсутствуют компьютерные программы, позволяющие моделировать такие распространенные в химической технологии и аналитической химии процессы, как растворение малорастворимого соединения в присутствии комплексообразователя или избытка осадителя. В связи с этим авторами был разработан алгоритм и компьютерная программа, использующая данный алгоритм (далее - программа), для моделирова- 
ния ионных равновесий при растворении одного или группы центральных ионов, находящихся в форме малорастворимых соединений с общим анионом (осаждающим анионом), в водном растворе соединения, способного переводить центральные ионы в раствор, (растворяющего лиганда) и при различных значениях ионной силы в растворе.

На основе оценки наиболее востребованных модельных расчетов для оптимизации гидрометаллургических технологий был сформулирован ряд упрощений, которые не лишают данные методы способности адекватно предсказывать поведение большинства указанных выше химических процессов, но позволяют сделать алгоритм настолько нетребовательным к вычислительной мощности, чтобы он мог быть мгновенно проведен на любом современном процессоре. Данные упрощения сформулированы в виде четырех требований к рассчитываемой равновесной системе и подробно описаны в разделе 1.1.

\section{1. Описание расчетного алгоритма}

\section{1. Назначение и возможности программы}

Программа реализована в среде MS Excel и Visual Basic for Applications для доработки и верификации разработанного математического алгоритма (интерфейс с примером готового расчета показан на рис. 1). Программа может применяться для разработки аналитических методик и оптимизации технологических процессов, сопровождающихся образованием малорастворимых соединений. Она позволяет определить ионный состав раствора при заданной начальной концентрации осаждающего реагента (или $\mathrm{pH}$ начала процесса) и растворяющего лиганда, а также ионную силу в растворе.

Указанная программа позволяет рассчитывать равновесный ионный состав раствора по двум алгоритмам:

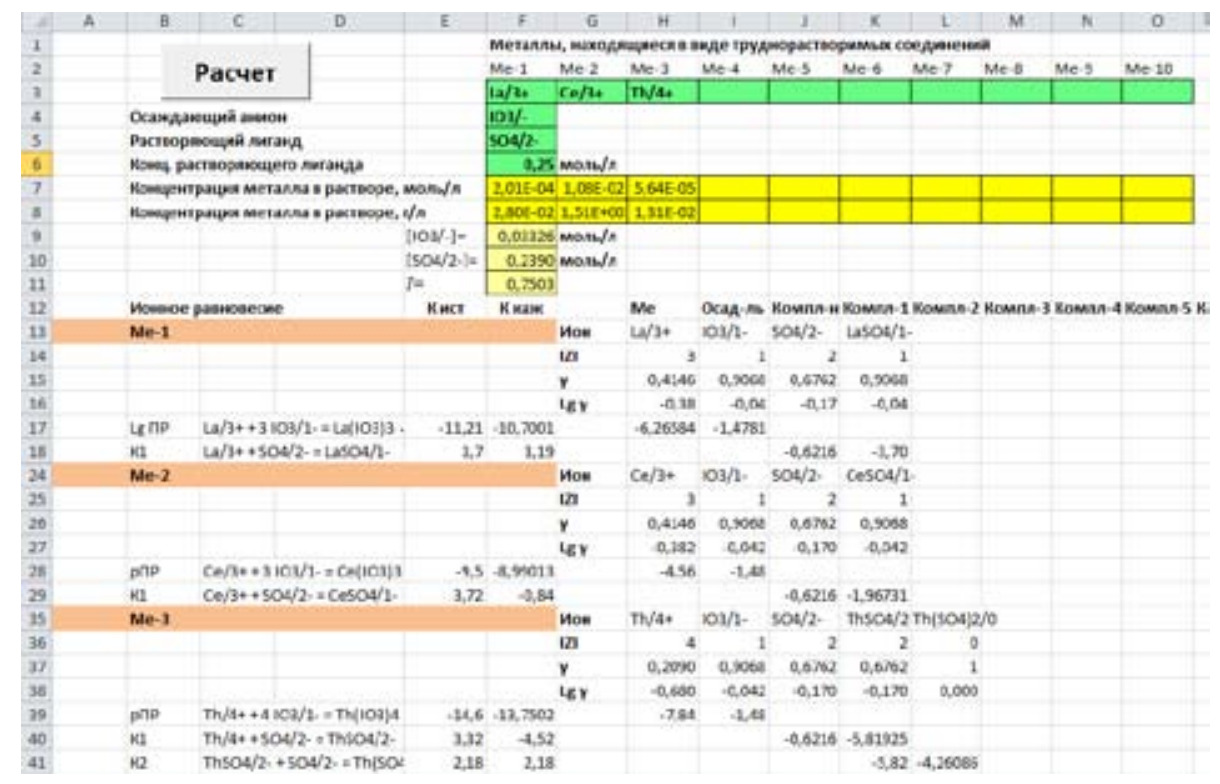

Рис. 1. Интерфейс программы с примером готового расчета

Fig. 1. The Program interface with an example of complete calculation 
Алгоритм 1 - растворение малорастворимой соли при заданной начальной концентрации растворяющего лиганда.

Алгоритм 2 - растворение малорастворимой соли в избытке осаждающего аниона (является частным случаем алгоритма 1).

Расчеты в программе проводятся на основе данных по произведениям растворимости (ПР, $K_{\mathrm{SP}}$ ) малорастворимых соединений, констант ионизации кислот и констант устойчивости комплексов, при расчетах также учитывается зависимость активности ионов от ионной силы в растворе.

Программа позволяет моделировать системы, которые удовлетворяют следующим четырем требованиям:

1) нахождение всех ионов металлов в равновесии с осадком;

2) все малорастворимые соединения образованы с одним общим анионом;

3) образование растворимых комплексов с осаждающим анионом протекает в незначительной степени (должно выполняться только при расчете по алгоритму 1);

4) процесс ведется при температуре, для которой определены константы равновесия (все используемые в программе константы определены при $\left.18-25^{\circ} \mathrm{C}\right)$.

Выполнение или невыполнение требования 1 не критично при расчетах для подавляющего большинства важных для технологических процессов химических равновесий ввиду того, что условия полного перехода иона в раствор могут быть определены при помощи простейшего материального баланса.

Требование 2 является справедливым для значительной доли технологических и природных малорастворимых соединений (пример - гидроксидные и оксалатные технологические осадки, монациты). Кроме того, при некоторой подготовке пользователя в области физической химии с помощью программы можно провести приближенный расчет для системы любой сложности, в том числе для содержащей несколько видов осаждающих ионов и несколько растворяющих лигандов.

Практически любой процесс растворения малорастворимых соединений в присутствии лигандов удовлетворяет требованию 3 ввиду того, что 1) константы образования (т. е. произведения растворимости) малорастворимых соединений практически всегда на несколько порядков больше, чем константы комплексообразования с теми же ионами в растворе, 2) при невысокой комплексообразующей способности растворяющего лиганда осаждающиеся центральные ионы и лиганд находятся в относительно низкой концентрации, соответственно, комплексообразование между ними незначительно, 3) при высокой комплексообразующей способности растворяющего лиганда все прочие реакции комплексообразования протекают в гораздо меньшей степени.

Требование 4 может быть устранено разложением констант ионных равновесий на их термодинамические составляющие $\left(\Delta H_{r}, \Delta S_{r}, C_{p}\right)$.

\section{2. Описание расчетного алгоритма}

В общем случае (алгоритм 1) ионные равновесия рассчитываются через циклические итерации в 3-мерном пространстве величин $I \supset[S] \supset[A]$, где:

$I$ - ионная сила в растворе; 
$[S]$ - концентрация в растворе осаждающего иона $(S)$, образующего с центральными ионами малорастворимые соединения, моль/л;

$[A]$ - концентрация в растворе свободной $(A)$ (не связанной в комплексы) комплексообразующей растворяющей частицы, моль/л.

Далее по тексту все величины, выражающие концентрации частиц, представляют собой молярные концентрации данных частиц (моль/л раствора).

При определении ионных равновесий в водном растворе точность расчетов в значительной степени зависит от учета влияния ионной силы на коэффициенты активности ионов ( $\gamma)$. Для незаряженных частиц в растворе $\gamma$ принимается равным 1. Для ионов $\gamma$ рассчитывается в программе по формуле Дэвис:

$$
\operatorname{Lg\gamma }=\left[\left(0,2 \cdot \sqrt{I}-\frac{0,511 \cdot \sqrt{I}}{1+1.5 \cdot \sqrt{I}}\right) \cdot Z^{2}\right],
$$

где $I$ - ионная сила раствора; $Z$ - заряд иона [4].

Итерация по I является высшей по уровню и включает в себя такую последовательность действий:

1*) Определение ионной силы $I$ по содержанию ионных форм в растворе, при этом:

если $\mathrm{n} 1$ = 1 , то комплексообразование и растворение металлов не учитывается;

если $\mathrm{n} l \neq 1$, то учитывается наличие всех ионных форм в растворе $(S$, комплексные ионы, индифферентный электролит), а полученный результат сравнивается с предыдущим результатом по формуле

$$
\sigma_{n 1}(I)=\left|1-\frac{I_{n 1}}{I_{n 1-1}}\right|,
$$

где $n l$ - счетчик итерации по $I ; \sigma_{n}(I)$ - относительная разница ионной силы на $n$ и $(n-1)$-той итерациях, если $\sigma_{n}(f)<0,2$, то за истинный результат принимается ионный состав, вычисленный по п. 5 на (n-1)-той итерации, и программа выходит из цикла.

$2 *)$ Расчет $\gamma$ для ионов при данной ионной силе по формуле 1.

$3^{*}$ ) Определение кажущихся констант равновесия $K($ каж.) при данной ионной силе по формуле

$$
K(\text { каж. })=K(\text { ист. }) \cdot \prod_{i=1}^{n} \gamma_{i}^{v i}
$$

где $K$ (каж.) - кажущаяся константа равновесия; $K\left(\right.$ ист.) - истинная константа равновесия; $\gamma_{i}-$ коэффициент активности $i$-той частицы, участвующей в равновесии; $v i$ - стехиометрический коэффициент $i$-той частицы, участвующей в равновесии.

$\left.4^{* *}\right)$ Равновесное изменение концентрации осаждающего аниона $\Delta \mathrm{C}(S)$ определяется следующим образом:

$\left.4.1^{*}\right)$ проверяется условие $n 2 \neq 1$ и условие $\sigma_{n 2}(S)<0,05$, если эти условия выполняются, то $\sigma_{n 2}(S)$ рассчитывается по формуле

$$
\sigma_{n 2}(S)=\left|1-\frac{\Delta C(S)_{n 2-1}}{\Delta \mathrm{C}_{S}(S)_{n 2-1}}\right|,
$$

где $n 2$ - счетчик итерация по $\mathrm{C}(S) ; \sigma_{n 2}(S)$ - относительная разница между $\Delta \mathrm{C}(S)$ и $\Delta \mathrm{C}_{S}(S)$; $\Delta \mathrm{C}(S)_{(\text {(n2-1) }}$ - увеличение концентрации осаждающего аниона, принятое на (n2-1)-той итерации; $\Delta \mathrm{C}_{\mathrm{S}}(S)_{(\mathrm{n} 2-1)}-$ фактическое изменение $\mathrm{C}(S)$, рассчитываемое по формуле 5 :

$$
-92-
$$




$$
\Delta C_{S}(S)_{(\mathrm{n} 2-1)}=\left(C_{A}-[A]\right) \cdot \frac{Z_{A}}{Z_{S}}
$$

где $C_{A}$ - исходная концентрация растворяющего лиганда, задаваемая пользователем; $[A]$ - равновесная концентрация свободного растворяющего лиганда, рассчитываемая по алгоритму, описанному в пп. 4.2-4.4; $Z_{A}$ - заряд растворяющего лиганда; $Z_{S}-$ заряд осаждающего аниона.

Если условия $n 2 \neq 1$ и $\sigma_{n 2}(S)<0,05$ выполняются, то программа выходит из цикла перебора по $n 2$, за истинный результат принимается ионный состав и $I$, рассчитанные по п. 4.6 на (n2-1)той итерации, и программа переходит к выполнению п. 1.

Если не выполняется хотя бы одно условие $\left(n 2 \neq 1\right.$ или $\left.\sigma_{n 2}(S)<0,05\right)$, то программа продолжает итерации по $n 2$, при этом изменение концентрации осаждающего аниона $\Delta \mathrm{C}(S)_{\text {(n2) }}$ задается следующим образом:

- если $\mathrm{n} 2=1$, то $\Delta \mathrm{C}(S)$ рассчитывается по формуле

$$
\Delta C(\mathrm{~S})_{(\mathrm{n} 2)}=0,1 \cdot C_{A} \cdot \frac{Z_{A}}{Z_{S}}
$$

- если $\mathrm{n} 2 \neq 1$ и $\Delta \mathrm{C}(S)_{(\mathrm{n} 2)}>\Delta \mathrm{C}_{\mathrm{S}}(S)_{(\mathrm{n} 2)}$, то $\Delta \mathrm{C}(S)$ рассчитывается по формуле

$$
\Delta C(S)_{(\mathrm{n} 2)}=0,5 \cdot \Delta C(S)_{(\mathrm{n} 2-1)},
$$

- если $\mathrm{n} 2 \neq 1$ и $\Delta \mathrm{C}(S)_{(\mathrm{n} 2)}<\Delta \mathrm{C}_{\mathrm{S}}(S)_{(\mathrm{n} 2)}$, то $\Delta \mathrm{C}(S)$ рассчитывается по формуле

$$
\Delta C(S)_{(\mathrm{n} 2)}=2 \cdot \Delta C(S)_{(\mathrm{n} 2-1)}
$$

$4.2 *)$ для каждого принятого значения $\Delta C(S)_{(\mathrm{n} 2)}$ и, соответственно, $[S]$ через произведение растворимости рассчитывается равновесная концентрация свободных ионов металлов $\mathrm{Me}^{\mathrm{n}+}$;

$4.3^{* *}$ ) рассчитывается равновесная концентрация [A], которая определяется через систему уравнений сопряженных равновесий комплексообразования и протонирования 7.

$$
\sum_{i=1}^{m 1} \sum_{n=1}^{m 2} \mathrm{~K}_{n}(\text { (каж. })_{i}=\frac{\left[M e A_{n}\right]}{\left[\operatorname{MeA}_{n-1}\right] \cdot[A]},
$$

где $m 1, m 2$ - количество центральных ионов и количество ступеней комплексообразования (протонирования) у $i$-того центрального иона соответственно.

Расчет $[A]$ производится путем последовательных приближений относительно $[A]$ до тех пор, пока $\sigma_{n 3}([A]) \leq 0,001$. Относительная погрешность $\sigma_{n 3}([A])$ определяется по формуле 8 :

$$
\sigma_{n 3}([A])=\left|1-\frac{\sum[A]_{n 3}}{C_{A}}\right|,
$$

где $n 3$ - счетчик итераций по $[A]$.

$\sum[A]_{n 3}$ - суммарная концентрация растворяющего лиганда, т. е. как свободного, так и связанного в различные комплексы и протонированные формы, рассчитываемая через систему уравнений 7.

При выполнении условия $\sigma_{n}([A]) \leq 0,001$ программа выходит из цикла и переходит к выполнению п. 4.4;

$\left.4.4^{*}\right)$ для $[A]$ через систему уравнений 7 определяется равновесный ионный состав по комплексам металлов;

$\left.4.5^{*}\right)$ для равновесного ионного состава раствора рассчитывается $I$;

$$
-93-
$$


$\left.4.6^{*}\right)$ для равновесного ионного состава раствора рассчитывается $\Delta \mathrm{C}_{\mathrm{S}}(S)_{(\mathrm{n} 2)}$ и $I$, затем начинается выполнение п. 4.1;

* - прямое вычисление величин аналитическим способом;

** - вычисление через итерации.

Существенным достоинством данного алгоритма является то, что число необходимых итераций не зависит от сложности формулы для расчета активности ионов. Более того, для повышения точности расчетов формулу 1 возможно заменить на группу формул или систему уравнений, а также включить уравнения для расчета активности воды, что также не приведет к значительному удлинению цепочки итераций.

При необходимости расчета систем, содержащих несколько растворяющих лигандов, и при необходимости учета изменения $\mathrm{pH}$ раствора возможно заменить 1-мерные итерации по $[A]$ на многомерные итерации, осуществляемые через итерации Ньютона, в данном случае мерность пространства $\mathrm{N}=$ количество растворяющих лигандов + 1 (итерации по оси $\left[\mathrm{H}^{+}\right]$или $\left.\left[\mathrm{OH}^{-}\right]\right)$.

\section{2. Результаты моделирования}

\section{1. Исходные данные для моделирования}

Для верификации разработанного алгоритма было проведено моделирование некоторых систем в водных растворах (11 систем), для которых имеются достоверные экспериментальные данные по растворимости. Верификация была проведена на следующих системах: $\mathrm{AgCl}-\mathrm{NaCl}$, $\mathrm{AgCl}-\mathrm{HCl}, \mathrm{AgCl}-\mathrm{HNO}_{3}, \mathrm{AgI}-\mathrm{HI}, \mathrm{Cu}(\mathrm{OH})_{2}-\mathrm{NaOH}, \mathrm{Zn}(\mathrm{OH})_{2}-\mathrm{NaOH}, \mathrm{Pb}(\mathrm{OH})_{2}-\mathrm{NaOH}, \mathrm{CaSO}_{4}-$ $\mathrm{NaNO}_{3}, \mathrm{SrSO}_{4}-\mathrm{NH}_{4} \mathrm{NO}_{3}, \mathrm{La}\left(\mathrm{IO}_{3}\right)_{3}-\mathrm{K}_{2} \mathrm{SO}_{4}, \mathrm{Ce}\left(\mathrm{IO}_{3}\right)_{3}-\mathrm{K}_{2} \mathrm{SO}_{4}$.

В разделах 2.2-2.5 приведена выборка результатов моделирования четырех систем. Константы ионных равновесий, которые использовались в данных расчетах, приведены в табл. 1. Для пересчета массовых концентраций в молярные для экспериментальных данных были использованы данные справочников [6, 7].

\section{2. Система $\mathrm{AgCl}-\mathrm{NaCl}$}

Система $\mathrm{AgCl}-\mathrm{NaCl}$ была смоделирована по алгоритму 2 (моделирование поведения малорастворимой соли в избытке осаждающего аниона). Моделирование растворимости $\mathrm{Ag}^{+}$в присутствии хлоридов было проведено для диапазона концентрации $\mathrm{NaCl}$ от 0,001 до 0,75 моль/л. Результаты теоретического расчета и экспериментальные данные при $\mathrm{t}=25^{\circ} \mathrm{C}$ приведены на рис. 2 .

Максимальное отклонение расчетных результатов в рассмотренном диапазоне концентраций относительно [5, табл. 3811] и [5, табл. 3812] составило 38 \%. Из графика на рис. 2 видно, что расчетное значение для концентрации серебра в водном растворе при $[\mathrm{NaCl}] \leq 0,25$ моль/л находится в пределах разброса экспериментальных значений.

При $[\mathrm{NaCl}]=0,25-0,75$ моль/л поведение теоретически рассчитанной кривой заметно отличается от экспериментальных кривых. Авторы считают, что подобное отклонение вызвано тем, что формула Дэвис (1) для расчета активности ионов, так же, как и другие подобные формулы (Дебая-Хюккеля, В. П. Васильева и др.), дает достаточно высокую погрешность при высоких $(I>0,1)$ значениях ионной силы. При этом погрешность расчета коэффициента активности иона тем выше, чем больше заряд этого иона. Так как при высоких концентрациях $\mathrm{Cl}^{-}$преобладают 
Таблица 1. Константы ионных равновесий, которые использовались в расчетах в разделах $2.2-2.5[4,5,6]$

Table 1. The ionic equilibria constants, which were used in the calculations in sections 2.2-2.5 [4, 5, 6]

\begin{tabular}{|c|c|}
\hline Уравнение реакции & Тип и величина константы равновесия \\
\hline 1 & 2 \\
\hline $\mathrm{Ag}^{+}+\mathrm{Cl}^{-}=\mathrm{AgCl} \downarrow$ & $\mathrm{p} \Pi P=9,75$ \\
\hline $\mathrm{Ag}^{+}+\mathrm{Cl}^{-}=\mathrm{AgCl}$ & $\mathrm{p} K_{l}=-3,04$ \\
\hline $\mathrm{AgCl}+\mathrm{Cl}^{-}=\mathrm{AgCl}_{2}{ }^{-}$ & $\mathrm{p} K_{2}=-2,00$ \\
\hline $\mathrm{AgCl}_{2}{ }^{-}+\mathrm{Cl}^{-}=\mathrm{AgCl}_{3}{ }^{2-}$ & $\mathrm{p} K_{3}=0,00$ \\
\hline $\mathrm{AgCl}_{3}{ }^{2-}+\mathrm{Cl}^{-}=\mathrm{AgCl}_{4}{ }^{3-}$ & $\mathrm{p} K_{4}=-0,26$ \\
\hline $\mathrm{Ag}^{+}+\mathrm{NO}_{3}{ }^{-}=\mathrm{AgNO}_{3}$ & $\mathrm{p} K_{l}=-0,29$ \\
\hline $\left.\mathrm{La}^{3+}+3 \mathrm{IO}_{3}{ }^{-}=\mathrm{La} \mathrm{IO}_{3}\right)_{3} \downarrow$ & $\mathrm{p} \Pi P=11,21$ \\
\hline $\mathrm{La}^{3+}+\mathrm{SO}_{4}{ }^{2-}=\mathrm{LaSO}_{4}{ }^{+}$ & $\mathrm{p} \Pi P=12,65 *$ \\
\hline $\mathrm{Ce}^{3+}+3 \mathrm{IO}_{3}{ }^{-}=\mathrm{Ce}^{2}\left(\mathrm{IO}_{3}\right)_{3} \downarrow$ & $\mathrm{p} K_{l}=-3,70$ \\
\hline $\mathrm{Ce}^{3+}+\mathrm{SO}_{4}{ }^{2-}=\mathrm{CeSO}_{4}{ }^{+}$ & $\mathrm{p} \Pi P=9,5$ \\
\hline $\mathrm{K}^{+}+\mathrm{SO}_{4}{ }^{2-}=\mathrm{KSO}_{4}{ }^{-}$ & $\mathrm{p} \Pi P=11,0 *$ \\
\hline $\mathrm{H}^{+}+\mathrm{HSO}_{4}{ }^{-}=\mathrm{H}_{2} \mathrm{SO}_{4}$ & $\mathrm{p} K_{l}=-3,72$ \\
\hline $\mathrm{H}^{+}+\mathrm{SO}_{4}{ }^{2-}=\mathrm{HSO}_{4}{ }^{-}$ & $\mathrm{p} K_{l}=-0,96$ \\
\hline $\mathrm{H}^{+}+\mathrm{IO}_{3}{ }^{-}=\mathrm{HIO}_{3}$ & $\mathrm{p} K_{a 1}=-3,0$ \\
\hline $\mathrm{p}$ & $\mathrm{p} K_{a 2}=1,9$ \\
\hline$K_{a}=0,77$ \\
\hline
\end{tabular}

* Значение не является справочным и рассчитано авторами по экспериментальным данным [5].

* The value is not a reference one and was calculated by the authors using the experimental data [5].

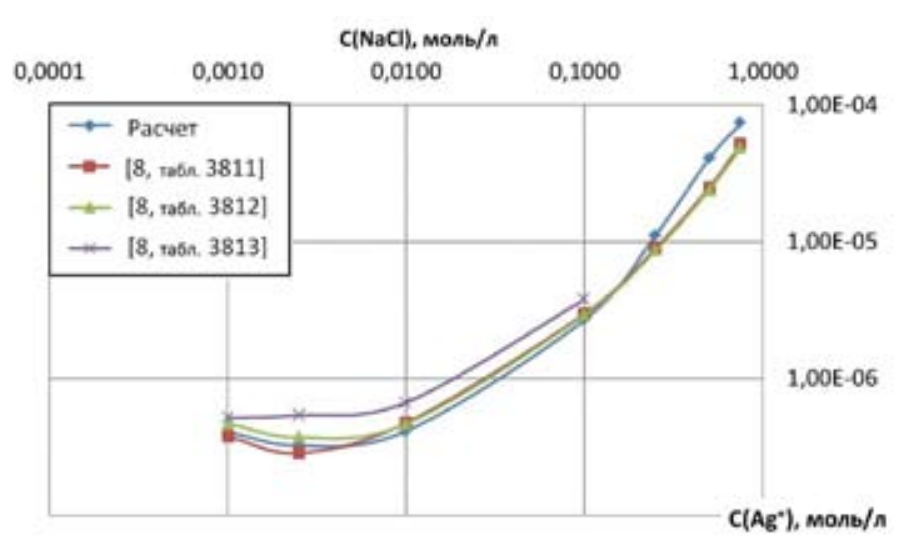

Рис. 2. Результаты теоретического расчета и экспериментальные данные по растворимости $\mathrm{AgCl}$ в присутствии $\mathrm{NaCl}$

Fig. 2. Results of the theoretical calculation and experimental data on the solubility of $\mathrm{AgCl}$ in the presence of $\mathrm{NaCl}$ 
комплексы с высоким зарядом $\left(\left[\mathrm{AgCl}_{3}\right]^{2-},\left[\mathrm{AgCl}_{4}\right]^{3-}\right)$, погрешность расчета активности ионов и других связанных с этим параметром величин становится еще более существенной.

\section{3. Система $\mathrm{AgCl}-\mathrm{HNO}_{3}$}

Система $\mathrm{AgCl}-\mathrm{HNO}_{3}$ была смоделирована по алгоритму 1 (моделирование растворения малорастворимого соединения в присутствии лиганда, образующего растворимые комплексы). Моделирование растворимости $\mathrm{Ag}^{+}$было проведено для диапазона концентрации $\mathrm{HNO}_{3}$ от 0 до 1,5 моль/л. Результаты теоретического расчета и экспериментальные данные при $\mathrm{t}=25{ }^{\circ} \mathrm{C}$ приведены на рис. 3.

Как видно из графика (рис. 3), экспериментальные кривые значительно отличаются друг от друга как по абсолютной величине, так и по форме. Как считают авторы, подобные отклонения вызваны различием в способах приготовления осадков $\mathrm{AgCl}$ (известно, что произведение растворимости некоторых труднорастворимых солей в значительной степени зависит от способа получения осадка и степени его гидратации, которая, в свою очередь, зависит от коэффициента активности воды в растворе) и присутствием в азотнокислом растворе примесей, например азотистой кислоты, которая способна образовываться в азотной кислоте под действием света и является гораздо более сильным комплексообразователем по отношению к ионам $\mathrm{Ag}^{+}$. Как видно из графика (рис. 3), расчетная кривая практически полностью лежит между экспериментальными кривыми.

\section{4. Система $\mathrm{La}\left(\mathrm{IO}_{3}\right)_{3}-\mathrm{K}_{2} \mathrm{SO}_{4}$}

Моделирование растворения $\mathrm{La}\left(\mathrm{IO}_{3}\right)_{3}$ в присутствии $\mathrm{SO}_{4}{ }^{2-}$ (алгоритм 1) было проведено для диапазона концентрации $\mathrm{K}_{2} \mathrm{SO}_{4}$ от $0,0002-0,1$ моль/л (экспериментальные данные до 0,05 моль/л). Результаты теоретического расчета и экспериментальные данные при $\mathrm{t}=25{ }^{\circ} \mathrm{C}$ приведены на рис. 4.

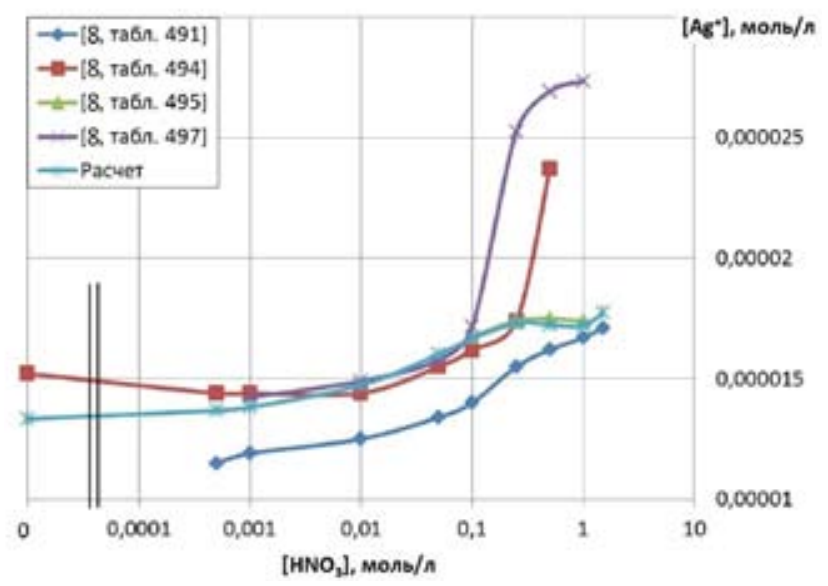

Рис. 3. Результаты теоретического расчета и экспериментальные данные по растворимости $\mathrm{AgCl} \mathrm{в}$ присутствии $\mathrm{HNO}_{3}$

Fig. 3. Results of the theoretical calculation and experimental data on the solubility of $\mathrm{AgCl}$ in the presence of $\mathrm{HNO}_{3}$ 


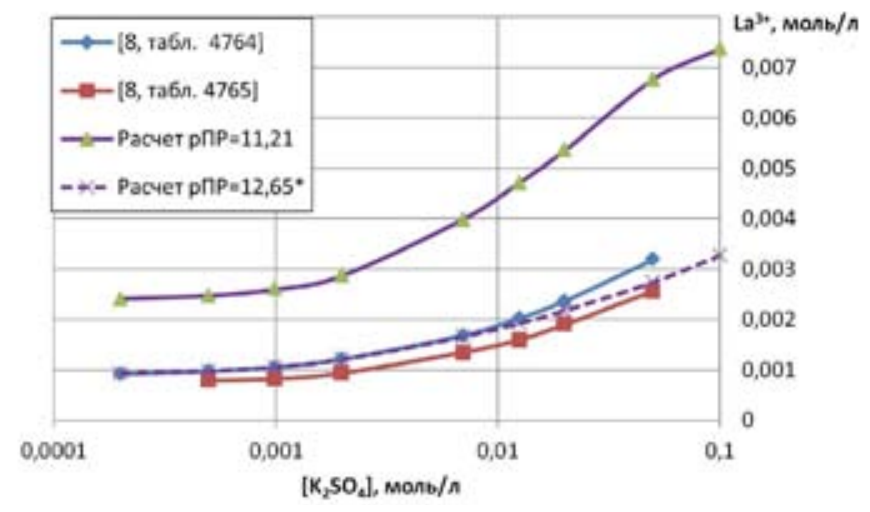

* Значение ПР $\left(\mathrm{La}^{3+}+3 \mathrm{IO}_{3}^{-}=\mathrm{La}\left(\mathrm{IO}_{3}\right)_{3} \downarrow\right)$ не является справочным и рассчитано авторами по экспериментальным данным $[5]$.

* The value of $K_{\mathrm{SP}}\left(\mathrm{La}^{3+}+3 \mathrm{IO}_{3}{ }^{-}=\mathrm{La}\left(\mathrm{IO}_{3}\right)_{3} \downarrow\right)$ is not a reference one and was calculated by the authors using the experimental data [5].

Рис. 4. Результаты теоретического расчета и экспериментальные данные по растворимости $\mathrm{La}\left(\mathrm{IO}_{3}\right)_{3}$ в присутствии $\mathrm{K}_{2} \mathrm{SO}_{4}$

Fig. 4. Results of the theoretical calculation and experimental data on the solubility of $\mathrm{La}_{(}\left(\mathrm{IO}_{3}\right)_{3}$ in the presence of $\mathrm{K}_{2} \mathrm{SO}_{4}$

Как видно из графика на рис. 4, полученная с помощью теоретического расчета для справочного значения ПР (рПР=11,21) растворимость несколько выше (в 2,1-2,6 раз относительно [5, табл. 4764]), чем экспериментальные результаты. Тем не менее в диапазоне, для которого были найдены экспериментальные результаты ([K $\left.\mathrm{SO}_{4}\right]=0,0002-0,05$ моль/л), формы теоретически рассчитанной и экспериментально определенной кривых растворимости практически идентичны, т. е. выполняется условие $y_{1}(\mathrm{x})=\mathrm{a} \cdot y_{2}(\mathrm{x})$, где a - константа, $y_{1}(\mathrm{x}), y_{2}(\mathrm{x})$ - функции, выражающие зависимость $\left[\mathrm{La}^{3+}\right](\equiv y)$ от $\left[\mathrm{K}_{2} \mathrm{SO}_{4}\right](\equiv x)$ для расчетных и экспериментальных данных.

Как было отмечено выше, произведение растворимости может сильно зависеть от способа получения осадка. В связи с этим было подобрано значение ПР $\left(\mathrm{La}^{3+}+3 \mathrm{IO}_{3}{ }^{-}=\mathrm{La}\left(\mathrm{IO}_{3}\right)_{3} \downarrow\right)$, при котором при $\left[\mathrm{K}_{2} \mathrm{SO}_{4}\right]=0,001$ моль/л теоретическая и экспериментальная растворимость совпадают. Данным способом было получено значение $\mathrm{p} \Pi P=12,65$, и для данного ПР была рассчитана растворимость при других концентрациях $\mathrm{K}_{2} \mathrm{SO}_{4}$. При $\left[\mathrm{K}_{2} \mathrm{SO}_{4}\right]<0,02$ моль/л теоретическая кривая растворимости практически совпала с экспериментальной, небольшое отклонение наблюдалось при $\left[\mathrm{K}_{2} \mathrm{SO}_{4}\right]>0,02$ и увеличивалось с ростом концентрации $\mathrm{K}_{2} \mathrm{SO}_{4}$. Авторы считают, что данное отклонение возникло из-за того, что не были учтены некоторые другие равновесия (данные по которым не были найдены), которые могут возникать при достаточно высоких концентрациях $\mathrm{IO}_{3}{ }^{-}$и $\mathrm{SO}_{4}{ }^{2-}$, и протекание которых может увеличивать растворимость La, например:

$$
\begin{aligned}
& \mathrm{LaSO}_{4}{ }^{+}+\mathrm{SO}_{4}{ }^{2-}=\mathrm{La}\left(\mathrm{SO}_{4}\right)_{2}{ }_{\text {раствор }} \\
& \mathrm{La}^{3+}+\mathrm{IO}_{3}{ }^{-}=\mathrm{LaIO}_{3}{ }^{2+} \text { раствор; } \\
& \mathrm{La}^{3+}+\mathrm{SO}_{4}{ }^{2-}+\mathrm{IO}_{3}=\mathrm{La}\left(\mathrm{SO}_{4}\right)\left(\mathrm{IO}_{3}\right)_{\text {раствор. }} .
\end{aligned}
$$

Стоит отметить, что изменение $K\left(\mathrm{La}^{3+}+\mathrm{SO}_{4}{ }^{2-}=\mathrm{LaSO}_{4}{ }^{+}\right)$без изменения ПР в расчетном алгоритме не приведет к тому, что теоретически рассчитанная кривая совпадет с экспери- 
ментальной. Данный факт легко объяснить с учетом сказанного выше - при увеличении или уменьшении $K$ кривая становится, соответственно, более крутой или пологой, при этом начинает нарушаться условие $y_{1}(\mathrm{x})=\mathrm{a} \cdot y_{2}(\mathrm{x})$, которое выполняется только при $\lg K \approx 3,7$ (использованное в расчетах справочное значение).

Учет сложных побочных равновесий, которые также способны повышать растворимость $\mathrm{La}\left(\mathrm{IO}_{3}\right)_{3}$, возможен, как это было указано в разделе 1.2 , после замены итерации по $[A]$ на $\mathrm{N}$-мерные итерации, осуществляемые через метод Ньютона. При этом для данной системы, а также для системы с множеством центральных ионов, $\mathrm{N}=2$ (без учета изменения $\mathrm{pH}$ ) и $\mathrm{N}=3$ (с учетом изменения $\mathrm{pH}$ ).

\section{5. Система $\mathrm{Ce}\left(\mathrm{IO}_{3}\right)_{3}-\mathrm{K}_{2} \mathrm{SO}_{4}$}

Моделирование растворения $\mathrm{Ce}\left(\mathrm{IO}_{3}\right)_{3}$ в присутствии $\mathrm{SO}_{4}{ }^{2-}$ (алгоритм 1) было проведено для диапазона концентрации $\mathrm{K}_{2} \mathrm{SO}_{4}$ от 0 до 0,1 моль/л. Результаты теоретического расчета и экспериментальные данные при $\mathrm{t}=25^{\circ} \mathrm{C}$ приведены на рис. 5 .

Как и в случае системы $\mathrm{La}\left(\mathrm{IO}_{3}\right)_{3}-\mathrm{K}_{2} \mathrm{SO}_{4}$, теоретически рассчитанная растворимость несколько выше полученной экспериментально. Также было подобрано значение ПР, при котором при $\left[\mathrm{K}_{2} \mathrm{SO}_{4}\right]=0,001$ теоретическая и экспериментальная растворимости совпадают. Было получено значение $\mathrm{p} \Pi P=11,0$ и рассчитана растворимость при других концентрациях $\mathrm{K}_{2} \mathrm{SO}_{4}$. При $\mathrm{p} П P=11,0$ теоретическая и экспериментальная кривые растворимости полностью совпали во всем рассматриваемом диапазоне. Авторы считают, что полное совпадение было получено благодаря отсутствию или протеканию в незначительной степени в данной системе, в отличие от аналогичной системы с $\mathrm{La}^{3+}$, других равновесий, кроме тех, которые были учтены при расчете.

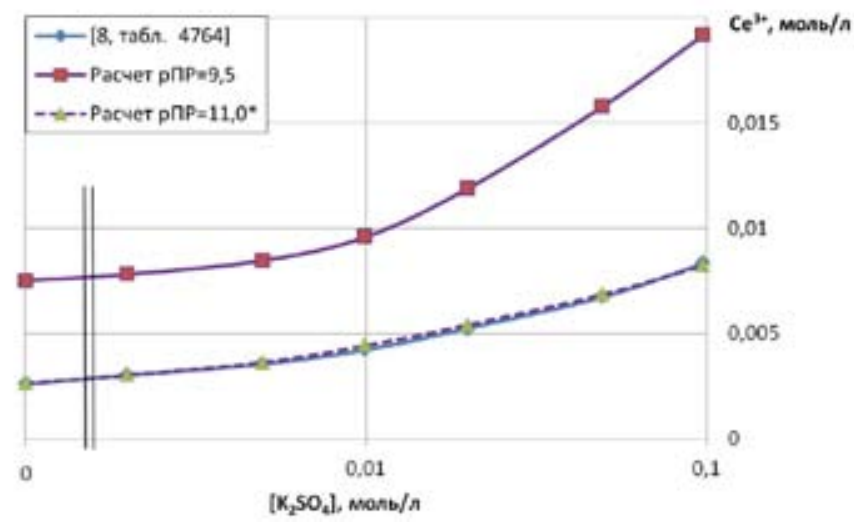

* Значение $П Р\left(\mathrm{Ce}^{3+}+3 \mathrm{IO}_{3}{ }^{-}=\mathrm{Ce}\left(\mathrm{IO}_{3}\right)_{3} \downarrow\right)$ не является справочным и рассчитано авторами по экспериментальным данным.

* The value of $K_{\mathrm{SP}}\left(\mathrm{Ce}^{3+}+3 \mathrm{IO}_{3}^{-}=\mathrm{Ce}\left(\mathrm{IO}_{3}\right)_{3} \downarrow\right)$ is not a reference one and was calculated by the authors using the experimental data.

Рис. 5. Результаты теоретического расчета и экспериментальные данные по растворимости $\mathrm{Ce}\left(\mathrm{IO}_{3}\right)_{3}$ в присутствии $\mathrm{K}_{2} \mathrm{SO}_{4}$

Fig. 5. Results of the theoretical calculation and experimental data on the solubility of $\mathrm{La}\left(\mathrm{IO}_{3}\right)_{3}$ in the presence of $\mathrm{K}_{2} \mathrm{SO}_{4}$ 


\section{Выводы}

1. На созданной демонстрационной компьютерной программе была проведена верификация алгоритма расчета растворения малорастворимых соединений в присутствии растворяющего лиганда или избытка осадителя, которая подтвердила адекватность созданного алгоритма при расчете систем, содержащих одно малорастворимое соединение.

2. Достоинства разработанного алгоритма:

- в расчеты может включаться любая формула (система уравнений, алгоритм) вычисления коэффициентов активности ионов и воды, при этом степень сложности используемой формулы (системы уравнений, алгоритма) незначительно влияет на количество требуемых итераций;

- незначительная зависимость количества требуемых итераций от количества малорастворимых соединений в системе;

- очень низкие требования к вычислительной мощности ЭВМ.

3. Недостаток разработанного алгоритма в том, что моделируемая система должна удовлетворять четырем требованиям, описанным в разделе 1.1 .

4. Был отмечен ряд проблем, которые, по мнению авторов, являются основными при моделировании гетерогенных систем вида «малорастворимая соль - раствор»:

- растворимость многих малорастворимых соединений в значительной степени зависит от способа получения осадка, что при неудачном выборе ПР для расчетной модели может приводить к заметной погрешности расчета (по результатам верификации разработанного алгоритма - до четырех раз), возможное решение данной проблемы - использовать в расчетных программах возможность коррекции ПР по экспериментальным данным, что было продемонстрировано выше;

- обобщенные формулы для расчета зависимости коэффициентов активности ионов от ионной силы в растворе могут давать заметную погрешность для ионов с высоким зарядом (по анализу справочных данных - до 2,2 раза);

- отсутствие в расчетном алгоритме некоторых реакций (например, реакций, по которым отсутствует литературная информация или которые не были учтены в расчетах ввиду относительно низких констант равновесия) может приводить к увеличению погрешности расчета при увеличении концентрации растворяющего лиганда (А), тем не менее данная погрешность меньше тех, которые были перечислены выше (по результатам верификации разработанного алгоритма - до $20 \%$ при $[\mathrm{A}] \leq 0,1$ моль/л); *

- для многих ионных равновесий и тем более для отдельных ионов отсутствуют данные по $\mathrm{KP}=\mathrm{f}(\mathrm{T}), \Delta \mathrm{HR}(\mathrm{Hf}), \Delta \mathrm{SR}(\mathrm{Sf}), \Delta \mathrm{CR}(\mathrm{Cp})$, что делает невозможным расчет для температур, значительно отличающихся от $25{ }^{\circ} \mathrm{C}$; *

- некоторые опубликованные экспериментальные данные получены разными исследователями с недостаточной воспроизводимостью, что затрудняет самостоятельное определение констант равновесия для малоизученных процессов.

* Проблемы также актуальны для всех прочих алгоритмов моделирования химических равновесий. 


\section{Список литературы}

1. Brinkley S.R. Calculation of the equilibrium composition of systems of many constituents. The Journal of Chemical Physics 1947. Vol. 15(2), P. 107-110.

2. Самарский А.А., Гулин А.В. Численные методы. М.: Наука, 1989. 432 с. [Samarskii A.A., Gulin A.V. Numerical methods. Moscow, Science, 1989. 432 p. (In Russ.)]

3. Демидович Б.П., Марон И.А. Основы вычислительной математики. М.: Физматгиз, 1966. 664 c. [Demidovich B.P., Maron I.A. Fundamentals of computational mathematics. Moscow, State public house of phys.-math. literature, 1966. 664 p. (In Russ.)]

4. Лурье Ю.Ю. Справочник по аналитической химии. М.: Химия, 1989. 448 с. [Lurie Yu.Yu. Handbook of Analytical Chemistry. Moscow, Chemistry, 1989. 448 p. (In Russ.)]

5. Кумок В.Н., Кулешова О.М., Карабин Л.А. Произведения растворимости. Н.: Наука, 1983. 267 c. [Kumok V.N., Kuleshova O.M., Karabin L.A. Solubility equilibria. Novosibirsk, Science, 1983. 267 p. (In Russ.)]

6. Никольский Б.П. Справочник химика. Том 3. Химическое равновесие и кинетика. Свойства растворов. Электродные процессы. М.: Химия, 1965. 1008 с. [Nikolsky B.P. Chemist reference book. Volume 3. Chemical equilibrium and kinetics. Properties of solutions. Electrode processes. Moscow, Chemistry, 1965. 1008 p. (In Russ.)]

7. Зайцев И.Д., Асеев Г.Г. Физико-химические свойства бинарных и многокомпонентных растворов неорганических веществ. Справочник. М.: Химия, 1988. 416 c. [Zaitsev I.D., Aseev G.G. Physical and chemical properties of binary and multi-component solutions of inorganic substances. Handbook. Moscow, Chemistry, 1988. 416 p. (In Russ.)]

8. Коган В.Б., Огородников С.К., Каффаров В.В. Справочник по растворимости. Том III. Тройные и многокомпонентные системы, образованные неорганическими веществами. Л.: Наука, 1969. 1171 c. [Kogan V.B., Ogorodnikov S.K., Kaffarov V.V. Reference book on solubility. Vol. III. Triple and multicomponent systems formed by inorganic substances. L., Science, 1969. 1171 p. (In Russ.)] 